Revue internationale P.M.E.

Économie et gestion de la petite et moyenne entreprise

\title{
Le comportement d'investissement en ressources technologiques externes et l'absorption technologique dans les moyennes entreprises industrielles
}

\section{Christian Le Bas}

Volume 6, numéro 2, 1993

URI : https://id.erudit.org/iderudit/1008209ar

DOI : https://doi.org/10.7202/1008209ar

Aller au sommaire du numéro

Éditeur(s)

Presses de l’Université du Québec

ISSN

0776-5436 (imprimé)

1918-9699 (numérique)

Découvrir la revue

Citer cet article

Le Bas, C. (1993). Le comportement d'investissement en ressources technologiques externes et l'absorption technologique dans les moyennes entreprises industrielles. Revue internationale P.M.E., 6(2), 35-64. https://doi.org/10.7202/1008209ar
Résumé de l'article

L'étude porte sur un échantillon de 20 entreprises industrielles de taille moyenne et a pour objet d'évaluer leur comportement quant à l'achat de ressources technologiques. Autrement dit, on est principalement concerné par l'absorption technologique conçue comme forme d'apprentissage. Les formes de l'acquisition externe de technologies diffèrent selon certaines caractéristiques sectorielles (trajectoire technologique...) ou structurelles (taille des firmes, capacité à structurer des fonctions). On montre finalement qu'il existe deux modèles d'apprentissage :

- un apprentissage complexe, mené par des entreprises plus (mieux) structurées, qui vont jusqu'à la sous-traitance de la R-D et construisent des liaisons avec des laboratoires. En termes d'apprentissage commercial, elles réalisent une bonne adéquation entre leurs technologies, leurs produits, leurs marchés.

- un apprentissage moins complexe (plus simple) mené par des entreprises qui apparaissent beaucoup moins structurées. En termes d'apprentissage technologique externe, ces entreprises ont un accès très particulier aux ressources technologiques externes, il passe exclusivement par l'achat d'équipement et de l'assistance technique.

On confirme également qu'un manque de relations technologiques denses (de type réseaux) peut diminuer l'efficacité des investissements technologiques des firmes. 


\title{
Le comportement d'investissement en ressources technologiques externes et l'absorption technologique dans les moyennes entreprises industrielles ${ }^{1}$
}

\author{
Christian LE BAS \\ Université Lumière Lyon $2^{*}$
}

\begin{abstract}
RÉSUMÉ
L'étude porte sur un échantillon de 20 entreprises industrielles de taille moyenne et a pour objet d'évaluer leur comportement quant à l'achat de ressources technologiques. Autrement dit, on est principalement concerné par l'absorption technologique conçue comme forme d'apprentissage. Les formes de l'acquisition externe de technologies diffèrent selon certaines caractéristiques sectorielles (trajectoire technologique...) ou structurelles (taille des firmes, capacité à structurer des fonctions). On montre finalement qu'il existe deux modèles d'apprentissage:

- un apprentissage complexe, mené par des entreprises plus (mieux) structurées, qui vont jusqu'à la sous-traitance de la $R-D$ et construisent des liaisons avec des laboratoires. En termes d'apprentissage commercial, elles réalisent une bonne adéquation entre leurs technologies, leurs produits, leurs marchés.
\end{abstract}

1. Cet article est issu d'une étude réalisée, au cours de l'année 1992, au sein de l'Unité de recherche appliquée Technologie, Entreprise, Système du Groupe ESC Lyon, en collaboration avec le Département animation économique et formation de la Chambre de commerce et d'industrie de Lyon et avec le soutien de la Direction générale XXIII de la Commission des communautés européennes. Je remercie Nathalie Michel qui a collaboré à l'étude, Isabelle Géniaux pour ses remarques judicieuses, Smaïl Ait El Hadj qui m'a prodigué ses conseils, ainsi que les rapporteurs de la revue.

* Christian Le Bas, professeur d'économie à l'Université Lumière Lyon 2 . Il est responsable de la Maîtrise en sciences économiques avec une spécialisation en Économie industrielle. Il dirige le Laboratoire d'économie et statistique appliquées et a publié plusieurs ouvrages ou articles sur l'économie des innovations et du changement technique. Adresse : Centre international de la recherche d'Écully, 93, Chemin des Mouilles, B.P. 58, 69132 Écully Cédex, France. 
- un apprentissage moins complexe (plus simple) mené par des entreprises qui apparaissent beaucoup moins structurées. En termes d'apprentissage technologique externe, ces entreprises ont un accès très particulier aux ressources technologiques externes, il passe exclusivement par l'achat d'équipement et de l'assistance technique.

On confirme également qu'un manque de relations technologiques denses (de type réseaux) peut diminuer l'efficacité des investissements technologiques des firmes.

\section{ABSTRACT}

This study treats a sample of 20 industrial middle-sized enterprises. It aims to evaluate their behaviour with regard to the buying of technological resources. We are mainly concerned by the technological absorption conceived as a way of learning. The forms of external technological acquisition may differ by sectoral characteristics (technological trajectory...), or by structural ones (size of the firms, capacity of organising functions). We are finally showing that there exist two different models of learning:

- a complex learning, practiced by more structured firms, who are even subcontracting their $R \& D$ and who are building up relationships with laboratories. In terms of commercial learning, they realise a good fit between their technologies, their products and their markets.

- a less complex learning (a more simplified one), practised by firms that seem much more unorganised. In terms of external learning, these enterprises have an access at external technological resources extremely particular: they learn only by buying equipment and by technical assistance.

We are also confirming that too few tight technological relationships (of the network type) might reduce the efficiency of technological investments made by firms.

\section{RESUMEN}

El estudio es hecho sobre un grupo de veinte firmas medianas y tiene par objetivo d'evaluar sus comportanvientos a la compra de capacidades tecnológicas.

Ademàs estamos principalmente implicados par el absorción tecnológico hecho como forma de aprentisage. Las formas de aquisición externas de tecnologias cambian par varias características sectoriales. (trayecto tecnológico...) a structurales (talla de las firmas, capacidad a structuar funciones). Constatamos finalmente que exsisten dos modelos de aprentisage:

- un aprentisage complejo, llavado par firmas mas structuradas, que van hasta la "sous traitance" de la $R-D$ y construyen la corespondencia con laboratorias. En terminas de aprentisage comercial, realizan una buena adecuación entre sus tecnologías, sus productos, sus mercados. 
- un aprentisage mas simple llevado par firmas que parecen muchomenos estructuradas. En termo de aprentisage tecnológico externo, estas firmas tienes une entrada muy particularmente a las bienes tecnológicos externos, pasa exclusivamente por la compra de material $y$ de asistencia tecnica.

Confirmamos también que una falta de relaciones tecnológicas espaciosas (de tipo "réseaux") puede desminuir la eficacidad de invertimientas tecnológicos de las firmas.

\section{Introduction}

On peut décrire l'histoire du management de la technologie de la firme comme une accumulation de savoir-faire, comme un apprentissage technologique (Dodgson, 1991). Toutefois, cet apprentissage a deux aspects : un apprentissage interne, la mobilisation des connaissances internes de la firme (et particulièrement - mais pas exclusivement - celles relevant du département de R-D) et un apprentissage externe, l'acquisition puis la maîtrise de connaissances externes. Apprendre des succès comme des échecs est une composante cruciale de la stratégie de l'entreprise, surtout lorsque l'environnement de la firme est mouvant. Les stratégies d'acquisition externe de technologies doivent être interprétées comme des éléments d'un processus d'apprentissage plus large. Un nombre croissant de PME se trouvent dans une situation d'évolution technique et économique qui engendre une demande technologique qu'elles ne sont pas en mesure de satisfaire par des moyens internes.

L'enquête innovation du SESSI, effectuée en France, offre une première description des investissements technologiques des entreprises industrielles, selon qu'il s'agisse d'acquisition interne de nouvelles technologies (R-D, études, dépôts de brevets) ou d'acquisition externe, soit sous forme immatérielle (acquisition du produit de la R-D, achats de brevets), soit sous forme matérielle (achats d'intrants technologiques). Les PME diversifient peu leurs sources d'acquisition (absorption) technique. Elles comptent beaucoup plus sur leur potentiel interne (bien qu'il soit plus faible que celui des grandes entreprises). Plus la taille de la firme croît, plus l'acquisition externe de technologies, sous sa forme immatérielle, est importante (par exemple, l'acquisition de brevet est tout à fait corrélé à la taille des firmes). On peut penser que le nombre de PME ayant recours à l'acquisition de R-D, et plus généralement de technologie externe, sera fortement croissant dans les années à venir. Par ailleurs, il est aujourd'hui admis que les firmes tentent de construire des liaisons de communications entre leur propre structure et celle des autres entreprises, voire d'en dériver des synergies (Dodgson, 1991). Ces stratégies de réseaux jouent un rôle décisif dans 
l'apprentissage technologique, puisqu'elles affectent la capacité d'absorption de la firme. Ainsi que le révèle un très remarquable travail réalisé par l'OCDE (1992), le développement des formes de type réseaux entre plusieurs firmes dans le domaine de la technologie renvoie fondamentalement à l'idée que les progrès techniques résultent très souvent d'échanges entre secteurs industriels (ou entre technologies), de fertilisation croisée, de transferts, de poussée d'une technologie (d'un secteur) sur une autre technologie (d'un autre secteur), de retombées de découvertes scientifiques sur de vastes champs de techniques. Quels facteurs commandent ce comportement d'investissement technologique externe?

Existe-t-il des différences qualitatives entre les entreprises moyennes et petites dans l'accès aux ressources externes ? Les structures des entreprises, les types de trajectoires technologiques constituent-ils des facteurs rendant compte de la diversité des comportements? Dans quelle mesure ce comportement d'investissement en ressources externes renvoie-t-il à une stratégie de réseaux ? Une évaluation s'impose, car elle permettrait de construire un diagnostic autour des politiques de soutien et d'actions qu'il faudrait privilégier pour répondre à la demande de développement technologique des entreprises.

On résume ici une recherche menée sur un échantillon d'entreprises industrielles moyennes (MEI), c'est-à-dire des entreprises réellement structurées, disposant de gammes de produits, et ayant une taille critique suffisante (autour d'un effectif de 200 personnes) pour investir dans la recherche-développement et le développement technologique. Ces entreprises se distinguent des petites entreprises (classiques ou de haute technologie ou encore dites innovantes) tout comme des entreprises beaucoup plus grandes (400 à 500 personnes). Les informations ont été recueillies par enquête directe auprès des entreprises.

\section{Cet article est structuré en quatre parties:}

1. Le thème de l'absorption et de l'apprentissage technologiques fondent la problématique de notre étude; il est défini en premier lieu.

2. Les caractéristiques de l'échantillon de moyennes entreprises industrielles (MEI) et la démarche de l'étude seront ensuite résumées.

3. Les résultats de l'enquête, c'est-à-dire les modes d'accès aux ressources technologiques externes et plus généralement le comportement d'absorption, retiendront notre attention.

4. En conclusion, quelques implications pour les stratégies des firmes et les politiques des États seront signalées. 
Source : EAE 1990 - SESSI

Champ : entreprises industrielles innovantes de 20 salariés et plus.

(1) Entreprises ayant recours à au moins un type de ressources internes.

(2) Entreprises ayant recours à au moins un type de ressources externes.

(3) Entreprises ayant recours à au moins un type d'intrants technologiques.

$\%$ en nombre d'entreprises

\begin{tabular}{|c|c|c|c|c|c|c|c|c|c|c|c|}
\hline \multirow[b]{2}{*}{$\begin{array}{l}\text { Tranches } \\
\text { de taille }\end{array}$} & \multicolumn{4}{|c|}{ SOURCES INTERNES } & \multicolumn{4}{|c|}{ SOURCES EXTENES } & \multicolumn{3}{|c|}{$\begin{array}{c}\text { INTRANTS } \\
\text { TECHNOLOGIQUES }\end{array}$} \\
\hline & $\begin{array}{l}\text { R-D } \\
\text { interne }\end{array}$ & Études & Brevets & (1) & $\begin{array}{l}\text { R-D du } \\
\text { groupe }\end{array}$ & $\begin{array}{l}\text { R-D } \\
\text { externe }\end{array}$ & $\begin{array}{l}\text { Droits/ } \\
\text { brevets }\end{array}$ & (2) & $\begin{array}{l}\text { Utilisation } \\
\text { novatrice } \\
\text { de biens } \\
\text { investis }\end{array}$ & $\begin{array}{l}\text { Matériaux } \\
\text { nouveaux }\end{array}$ & (3) \\
\hline $20-49$ & 40,0 & 70,9 & 20,0 & 79,4 & 18,4 & 42,6 & 17,0 & 56,0 & 71,7 & 57,5 & 82,8 \\
\hline $50-99$ & 51,5 & 78,7 & 27,8 & 87,6 & 28,2 & 45,7 & 20,1 & 63,7 & 72,8 & 59,4 & 83,1 \\
\hline $100-199$ & 61,8 & 85,6 & 36,0 & 92,6 & 37,5 & 47,9 & 22,2 & 69,6 & 78,9 & 68,2 & 88,5 \\
\hline $200-499$ & 72,2 & 86,7 & 43,7 & 94,9 & 45,9 & 53,5 & 31,4 & 76,6 & 81,3 & 65,6 & 89,5 \\
\hline $500-999$ & 80,5 & 90,4 & 58,4 & 97,1 & 69,2 & 60,6 & 39,4 & 85,8 & 85,8 & 75,7 & 93,4 \\
\hline $1000-1999$ & 89,4 & 92,6 & 70,7 & 97,9 & 69,7 & 62,8 & 40,4 & 88,8 & 89,4 & 78,2 & 94,1 \\
\hline 2000 et $t$ & 91,4 & 95,3 & 76,6 & 99,2 & 78,1 & 71,9 & 55,5 & 96,9 & 93,0 & 88,3 & 96,9 \\
\hline TOTAL & 50,3 & 76,7 & 28,1 & 85,1 & 28,1 & 45,9 & 20,9 & 62,8 & 74,4 & 60,9 & 84,6 \\
\hline
\end{tabular}




\section{Absorption et apprentissage technologiques: une problématique centrale ${ }^{2}$}

\subsection{Apprentissage interne et apprentissage externe de la firme}

Qu'implique le fait que la firme ne peut pas être considérée comme un «point», un vecteur technologique, mais qu'elle a une réelle épaisseur, celle d'une organisation? L'organisation est structurée en services, départements, unités, lesquels remplissent des fonctions différentes ou se situent à des stades différents de la chaîne logistique d'une fonction. Dans chacun des sous-systèmes, il y a des processus d'apprentissage par la pratique, des apprentissages plus complexes. Il est trivial aujourd'hui de reconnaître que la recherche-développement est aussi un processus d'apprentissage (Rosenberg, 1982). Si la firme est une organisation, il s'ensuit que l'apprentissage est nécessairement social ou partagé (shared-learning, Adler, 1990) d'où l'importance des interfaces entre les différentes fonctions, de la circulation des informations entre les différents départements. R.M. Cyert et J.G. March avaient naguère défini une théorie des règles de conduite de la firme. En matière de règle de décision, les entreprises adoptent des routines et, sur ces bases, les entreprises choisissent en fonction des informations nouvelles tirées de l'environnement d'autres actions ou une modulation des anciennes. Toutefois, elles peuvent également, en fonction des résultats acquis sur l'efficacité des routines passées, changer de routines. Il s'agit là d'un apprentissage "supérieur» et adaptatif par lequel la firme acquiert une nouvelle adaptabilité. Cette distinction est reprise par Argyris et Schön (1978), lesquels définissent le premier comme une rétroaction simple (single-loop learning) et le second comme une rétroaction redoublée (doubleloop learning). On peut y retrouver l'opposition entre un apprentissage tactique et un apprentissage stratégique (Dodgson, 1991).

On peut évaluer les processus d'apprentissage en retenant deux «formes idéales »: l'apprentissage technologique interne et l'apprentissage technologique externe. Il s'agit d'une distinction aujourd'hui admise dans la littérature, y compris celle qui situe son analyse autour des stratégies des firmes (Dodgson, 1991). Elle est commode et réaliste, encore qu'il faille bien en mesurer la faible charge réductrice. Il n'existe pas de firmes qui, de façon durable, auraient recours à un apprentissage purement interne; même si des firmes ne réalisent pas d'investissements dans des ressources technologiques externes (biens d'équipements, brevets, licences), elles exploitent toujours des informations techniques et scientifiques qu'elles n'ont pas produites, elles embauchent des salariés

2. On se référera également à C. Le Bas et E. Zuscovitch (1993). 
nouveaux possédant des connaissances ou des compétences spécifiques, etc. Seules, peut-être, des petites entreprises n'échangent pas avec l'extérieur, le prototype étant la cellule de production artisanale; mais il s'agit aussi d'une cellule parmi les plus conservatrices en termes de technologies, car elle repose sur un savoir-faire immuable. Autrement dit, il y a toujours acquisitions de ressources ou de connaissances techniques externes (et donc un apprentissage externe). On peut éventuellement aller plus loin et reconnaître (le cas du savoirfaire de l'artisan est de ce point de vue extrêmement illustrant mais en creux) qu'un effort extérieur est toujours nécessaire à des progrès significatifs, un transfert extérieur autorisant la levée d'un blocage technique. Symétriquement, il n'y a pas d'apprentissage externe sans apprentissage interne : la capacité d'assimiler les informations ou les ressources techniques extérieures dépend des compétences et des capacités internes. Quel intérêt peut alors présenter cette distinction entre les deux « formes » d'apprentissage alors qu'il y a toujours un mélange entre les deux? En premier lieu, il nous semble qu'elle fonde une typologie féconde des stratégies ou des comportements des firmes devant l'innovation. Ainsi, la firme innovante qui produit de la technologie à travers son activité de R-D ou la gestion des effets induits d'un département à un autre relève plutôt d'une logique d'apprentissage interne (avec évidemment les réserves déjà émises), alors que la firme imitatrice (suiveuse) expérimente une autre logique de changement technique et donc d'apprentissage de type principalement externe. Les premiers schémas de la diffusion de l'innovation, largement dominés par des représentations empiriques, dissociaient plusieurs catégories de comportement d'imitation à partir d'une échelle «de retard » (premiers retardataires, retardataires suiveurs...) et donc autant de processus d'apprentissage. Toutefois, l'imitation n'est jamais un processus de « pure » imitation; il y a toujours de l'innovation dans un tel processus. En cela, l'imitation n'est pas une reproduction stricte (répétition). Le second intérêt réside dans des considérations plus didactiques. L'apprentissage externe met en jeu d'autres mécanismes, fait appel à d'autres configurations systémiques; ainsi, il peut être étudié séparément. On veut souligner ici qu'à un niveau d'abstraction élevé, tout apprentissage technologique peut être décrit comme une séquence recherche-accumulation-appréciation (Dodgson, 1991). Autrement dit, il faut rechercher de l'information, l'incorporer dans le bloc des actifs technologiques de la firme (processus d'accumulation ou de mémorisation) ou dans ses compétences économiques (Carlson et Stankiewicz, 1991), l'évaluer (apprendre des erreurs). On suggère plutôt ici que l'appréciation (ou l'évaluation) opère comme une force de rappel à chaque stade élémentaire de la séquence. Par rapport au schéma de M. Dodgson (1991), on a ajouté le résultat (l'accroissement des performances de la firme): sous l'effet de l'apprentissage, l'organisation progresse ; en apprenant, elle accroît son efficience. 
Schéma général de l'apprentissage adapté de M. Dodgson (1991)

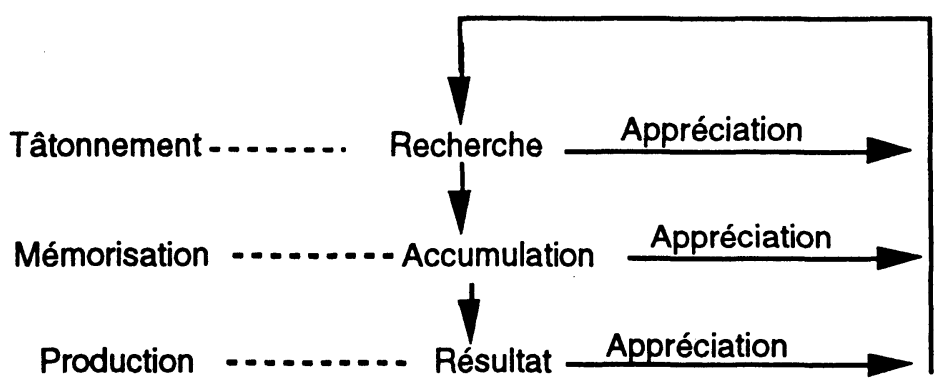

\section{L'apprentissage externe}

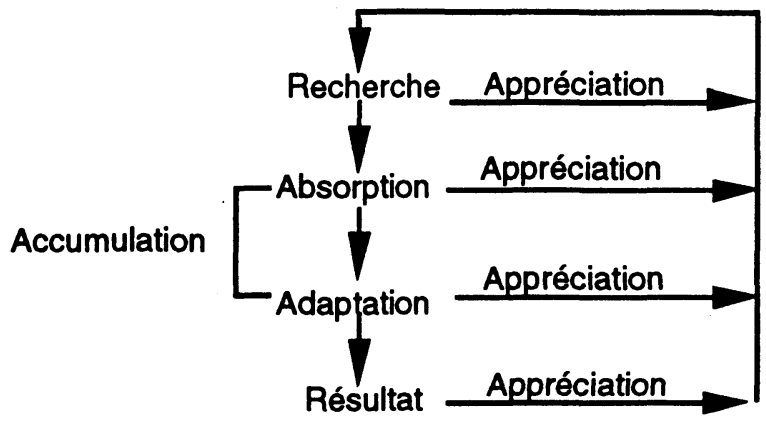

L'apprentissage interne implique la recherche de connaissances, de savoirfaire. L'organisation apprend à travers ses activités propres. On peut disposer d'autant de schémas d'apprentissage que de modèles d'organisation des fonctions de la firme ou des départements. Bien évidemment, un éclairage très riche pour l'économiste de l'innovation tient dans la constitution des apprentissages selon les propres champs technologiques de la firme. L'apprentissage externe met en jeu d'autres mécanismes. La phase d'accumulation est nécessairement plus riche. Sous l'influence des travaux récents, on peut l'interpréter comme étant avant tout un processus d'absorption par l'organisation d'informations venant de l'environnement. Le terme se trouve bien choisi; il décrit bien le fait que l'organisation se nourrit (grossit) à partir de ponctions sur son environnement. Le processus d'adaptation (Radnor, 1991), quant à lui, rend compte des formes que prend l'incorporation des ressources technologiques acquises à la structure présente du capital technologique de la firme. 
Il est particulièrement instructif de retenir comme éclairage les différents facteurs déterminant des processus d'apprentissage plus efficients, supportant des coûts moindres, ou procurant des gains d'efficacité plus élevés. Dans la littérature récente, l'étude de l'apprentissage interne a été renouvelé en isolant plusieurs modes de coordination internes à la firme. Avec les différents leviers de la créativité dans l'activité de R-D, ils forment les facteurs impulsant un «bon apprentissage interne». Quant à l'apprentissage externe, il a été étudié en référence avec la capacité d'absorption de la firme (Cohen et Levinthal, 1990).

\subsection{Absorption technologique et apprentissage externe}

Dans un article récent, W.M. Cohen et D.A. Levinthal (1990) proposent un schéma rendant compte de la capacité à exploiter les connaissances technologiques comme composante fondamentale de la capacité d'innovation de la firme. Cette capacité à percevoir, trier et utiliser les connaissances externes à la firme est également fonction du niveau technologique atteint par la firme ou de sa capacité à maîtriser les corps de savoirs fondamentaux. En recourant à une vision dynamique ou, ce qui revient au même, en introduisant le temps, on peut avancer que les savoirs acquis antérieurement par la firme déterminent la capacité d'absorption de la période.

Ces connaissances relèvent:

- des compétences de base, générales ou techniques,

- des connaissances scientifiques et technologiques les plus récentes de différents champs,

- des capacités à communiquer (shared language) au sein de la firme.

Cette capacité collectivement constituée permet de reconnaître la valeur des nouvelles informations, de les assimiler, de les appliquer à un nouveau projet industriel viable commercialement.

La capacité d'absorption constitue donc un concept visant à dialectiser le processus de création technologique : la firme doit combiner ce qu'elle peut prendre à l'extérieur (dans son environnement) avec son propre capital technologique (interne), afin de mener à bien le processus innovatif. L'interactivité au sein de cette unité est forte: puisque ce que la firme peut assimiler (donc venant de l'extérieur) est assez fortement contraint par son savoir antérieur accumulé. Mais c'est également le savoir acquis à l'extérieur qui, à travers un processus de stratification et de mémorisation, a enrichi les savoirs propres, originels de la firme. Nous nous limitons ici à mieux définir la capacité d'absorption. 
La capacité d'absorption d'une firme dépend de la capacité d'absorption de ses membres. Cette notion de capacité individuelle est particulièrement développée dans les sciences de l'éducation, du comportement social et de la psychologie. Une prédiction essentielle serait que le savoir accumulé autorise l'apprentissage d'une nouvelle connaissance propre ou parente, propriété également valide lorsqu'il s'agit d'un corps de savoirs (d'un ensemble structuré de savoirs). Il y a même une possibilité de transférer des capacités d'apprentissage sur des corps de savoir-faire apparemment différents, mais organisés de façon similaire; en bref, des corps de savoirs reliés par une forte proximité.

Venons-en maintenant à la capacité d'absorption collective, la capacité de la firme. Elle dépend explicitement de la capacité et du savoir-faire de ses membres, mais également de la façon dont ils sont organisés: la capacité de l'organisation ne dépend pas seulement de la structuration des interfaces entre la firme et son environnement, mais aussi des transferts de connaissances entre et dans les sous-ensembles (ou divisions internes) de la firme. Ici, la théorie de la capacité d'absorption rejoint les analyses des structures de communication interne à la firme (Katz et Kahn, 1966).

Comment définir la capacité d'absorption de l'organisation? Cohen et Levinthal (1990) suggèrent qu'elle repose:

- sur les capacités de chaque membre ;

- sur la capacité de recueillir l'information de l'environnement (I) et de transmettre les informations entre les départements de la firme (II) ;

(Dans cette mesure, les fonctions d'interfaces (entre l'entreprise et l'environnement, comme dans I, et entre les départements de l'unité de production, comme dans II) sont cruciales pour «optimiser» l'exploitation des informations: ce point est effectivement abordé dans les études portant, par exemple, sur la veille technologique.)

- sur la nature et la distribution de la qualification dans l'organisation.

Il devient clair que la capacité d'absorption de la firme repose largement sur son modèle d'organisation. Il y a des schémas d'organisation qui dynamisent les capacités individuelles. Dans cette perspective, d'intéressantes prédictions ressortent.

- Une opposition semble exister entre l'efficience de la communication interne à la firme et la capacité de chacune des unités (ou départements) de la firme à assimiler les informations des autres unités ou de l'environnement extérieur. Si un aspect est dominant (par rapport au second), il s'ensuit un réel dysfonctionnement. Ce marchandage vient du fait que 
la communication interne à la firme sera bonne si ses membres partagent la même culture. La ponction sur l'environnement de l'information technique et son traitement seront efficaces seulement si la firme dispose d'une certaine diversité de savoirs.

- Des fonctions d'interfaces, transversales, affectent positivement la capacité d'absorption et les performances innovatives. Une grande partie de la littérature souligne l'importance des interdépendances entre les fonctions (les départements) de R-D, design, fabrication, marketing (Mansfield, 1968; Rosenberg et Kline, 1986) et a même fait l'objet de comparaisons internationales aux prédictions fort instructives quant à la «supériorité» de la firme japonaise (Aoki, 1990). Toutefois, si les liaisons fortes accroissent la productivité, leurs effets peuvent être plus ambigus et tendraient à réduire la capacité d'absorption. Cohen et Levinthal avancent qu'une fonction nécessairement transversale (a cross function) d'absorption comprendrait la gestion des liaisons entre une unité de R-D et l'ensemble de la firme.

- Si la capacité d'absorption est largement interne, les firmes ont-elles les moyens d'acheter à l'extérieur une partie de cette capacité (embauche de personnel, contrats avec des sociétés de consultants, «rachats éducatifs»)? Sur ce point, Cohen et Levinthal (1990) avancent que ceci est assez illusoire: toute firme doit avoir une taille critique minimum de capacité technologique interne (sur les produits et les procédés) et l'intégration de nouvelles personnes, ainsi que l'émergence de nouvelles routines (Nelson et Winter, 1982) dans les activités de recherche demandent du temps. Plus récemment, M. Radnor (1991) a montré que la perspective du «faire ou du faire faire» n'éclaire que très pauvrement les stratégies d'acquisition technologique. On s'accorde ainsi à reconnaître que les voies d'accès à de nouvelles technologies ont pour base un mélange entre la production interne et l'acquisition externe. La firme doit adapter (Radnor, 1991) ce qu'elle achète pour en tirer l'efficacité maximum, cette adaptation (qui est en quelque sorte un autre nom pour l'apprentissage) n'est possible que si la firme dispose de compétences internes fortes.

- En termes dynamiques, le savoir antérieur doit être fortement relié au nouveau savoir de manière à faciliter l'assimilation de ce dernier. Ainsi, deux propriétés de la capacité d'absorption apparaissent: son caractère cumulatif et son rôle crucial dans la formation des anticipations quant à l'évolution des technologies. 


\section{Un échantillon de moyennes entreprises industrielles (MEI) aux structures diversifiées}

\subsection{Un échantillon de moyennes entreprises industrielles}

Le champ de l'enquête qui s'est déroulée entre février et juin 1992 concerne des PME ayant un effectif situé autour de 200 salariés, y compris les filiales de groupes, dans trois secteurs industriels (textile, mécanique et électronique). Sur une soixantaine d'entreprises contactées en région Rhône-Alpes, seulement vingt-cinq ont accepté de nous rencontrer et de répondre à un questionnaire au cours d'un entretien dont la durée variait entre 30 minutes et 2 heures. Il est indéniable que la qualité des informations recueillies est fonction du temps passé avec l'interlocuteur, ainsi que du poste qu'occupe ce dernier (chef d'entreprise, responsable du bureau d'étude, du service recherche-développement...). Finalement, nous avons retiré de l'échantillon les firmes aux effectifs trop importants et celles relevant d'un groupe industriel.

\subsubsection{Méthodologie de l'enquête}

Les listes d'entreprises, pour les secteurs textile et mécanique, nous ont été fournies par les centres techniques correspondants (ITF et CETIM). Ces organismes publics consacrent chaque année plus d'un milliard de francs à la recherche appliquée, la veille technologique et l'information. Ils sont chargés de favoriser les transferts de technologies vers l'ensemble des entreprises d'une profession. Nous n'avons donc pas procédé à une sélection aléatoire d'entreprises. Nous nous en sommes tenus exclusivement, pour les secteurs mécanique et textile, aux listes de ces centres techniques. Nous pensions ainsi rencontrer des PME actives, tournées vers des problèmes d'acquisition de technologies et ouvertes à une politique de réseaux, étant déjà en relation avec des centres techniques, instruments de coopération industrielle. De ce fait, l'échantillon d'entreprises enquêtées ne peut prétendre être représentatif des stratégies d'acquisitions technologiques externes des PME. Nous avons étudié une population d'entreprises moyennes qui ont des rapports avec des centres techniques, ce qui témoigne de l'existence d'une gestion réelle de la technologie, et qui n'est pas le cas pour toutes les PME (Le Bas, 1989).

La sélection de la population dans le secteur électronique a été différente dans le sens où nous ne connaissons pas de centre technique propre à ce secteur. C'est pourquoi nous avons recherché un organisme qui était en étroite relation avec des entreprises du secteur. La SCTT (Société de France Télécom) nous a présenté une liste de PME qui pouvaient répondre à notre attente. De cette liste, trois ont participé à notre étude. Il convient d'ores et déjà d'indiquer que ces entreprises de la construction électronique ont une taille (mesurée par effectif) 
très faible, plus faible que la moyenne des autres. Ceci provient du fait que dans ce secteur, les moyennes entreprises (200 à 400 personnes) font souvent partie d'un groupe industriel et que nous avons renoncé à étudier le management de la technologie dans ce type de firme.

\subsubsection{Profil des PME enquêtées}

Nous retrouvons dans notre groupe trois des cinq types de PME identifiés par Julien et Marchesnay (1992): les petites entreprises des secteurs traditionnels, les moyennes entreprises à organisation décentralisée et direction instruite et les entreprises à production spécifique. Toutes les entreprises enquêtées ont moins de 300 salariés sauf une qui a plus de 800 employés, laquelle n'a d'ailleurs pas été exploitée dans notre champ d'étude. Leur chiffre d'affaires varie de 15 millions à 470 millions de francs :

- 9 entreprises appartiennent au secteur mécanique, avec une taille moyenne de 230 salariés;

- 5 entreprises appartiennent au secteur textile, avec une taille moyenne de 240 salariés;

- 4 entreprises appartiennent au secteur électronique, dont 3 ont moins de 50 employés (en moyenne 45), et une ayant 100 salariés.

\section{Les caractéristiques de l'échantillon de MEI}

Taille moyenne (effectif) moyenne R-D/CA

Variété sectorielle

Entreprises possédant un laboratoire

Entreprises possédant un service de recherche
201

2 entreprises ont un effectif supérieur à 400 personnes,

7 entreprises ont un effectif compris entre 200 et 300 personnes

5 entreprises ont un effectif compris entre 100 et 200 personnes

3 entreprises ont un effectif inférieur à 50 personnes

$3,0 \%$

6 entreprises du textile

7 entreprises de la mécanique

4 entreprises de l'électronique

$50 \%$

$50 \%$ 
Si le degré d'intensité technologique moyen de la population est de $3 \%$, cet indicateur connaît une grande dispersion suivant les entreprises. On peut noter que les entreprises qui ont une intensité technologique forte (plus de $3 \%$ ) ont un chiffre d'affaires plutôt élevé.

Pour ce qui est de la structuration des fonctions de conception et de recherche, $65 \%$ des entreprises ont un bureau d'étude, $53 \%$ ont institutionnalisé un service de recherche et $30 \%$ seulement ont un laboratoire.

\begin{tabular}{ccccc}
\hline & $\begin{array}{c}\text { Bureau } \\
\text { d'étude }\end{array}$ & $\begin{array}{c}\text { Bureau } \\
\text { des méthodes }\end{array}$ & $\begin{array}{c}\text { Service } \\
\text { de recherche }\end{array}$ & Laboratoire \\
\hline Population totale & 11 & 10 & 9 & 5 \\
\hline en $\%$ & $65 \%$ & $59 \%$ & $53 \%$ & $30 \%$ \\
\hline
\end{tabular}

Il reste que, compte tenu de la taille de l'échantillon, il serait vain de vouloir recourir à des méthodes de validation statistique. Toutefois, des prédictions ressortent.

\subsection{Trois secteurs, mais plusieurs trajectoires technologiques}

La nature des opportunités technologiques et des menaces auxquelles les firmes doivent faire face est largement déterminée par l'activité principale de l'entreprise et les caractéristiques structurelles des secteurs. Si l'on désire construire un descriptif des capacités des secteurs à générer, entretenir et diffuser le changement technique, il est particulièrement tentant de les fonder sur l'origine du changement technique.

On peut en effet retenir un premier axe associé aux sources de la nouveauté technique: progrès scientifiques, savoir-faire techniques, progrès de routine, etc., et un second relatif au lieu: le progrès est-il issu du secteur ou du dehors?

Ces deux axes fondent en réalité la taxinomie suggérée par K. Pavitt (1984). Quatre trajectoires technologiques sont définies:

- Les secteurs acquérant l'innovation auprès d'offreurs dominants (supplier-dominated). Les firmes de ce secteur ne génèrent pas l'innovation, elles l'acquièrent par l'intermédiaire de l'achat de biens d'équipement (innovation de procédés) issus de secteurs où domine plutôt un pouvoir de monopole. K. Pavitt classe dans cette catégorie les vieilles industries de biens de consommation (textile, habillement, bois...). 
- Les secteurs à rendements croissants à l'échelle (scale-intensive). Les technologies utilisées par ces firmes sont en général complexes. Elles sont très concentrées et, à la différence de celles de la première trajectoire, disposent de département de R-D. Les innovations qu'elles supportent touchent à la fois le procédé et les produits. Les industries de biens intermédiaires, la métallurgie et les transports sont représentatives de ces conditions.

- Les secteurs renouvelant leur technologie par des poussées scientifiques (science-based). Dans ces industries, les innovations (qui portent beaucoup sur les produits) sont issues des découvertes de laboratoires. Ces secteurs ont de façon systématique, et depuis la fin du XIX $\mathrm{Xiècle,}^{\mathrm{e}}$. conçu leur développement sur les avancées des recherches fondamentales. On y retrouve la chimie et la pharmacie.

- Les secteurs à offreurs spécialisés (specialised suppliers ). Les firmes, ici, sont très petites, fabriquant des biens à contenu technologique élevé. Elles réalisent des produits relevant de la haute technologie : instruments scientifiques et ingénierie.

D'après nous, il y a lieu de rajouter à cette typologie une autre catégorie importante qui s'inscrit bien dans notre démarche poursuivie jusqu'ici. Il s'agit des secteurs dont le développement technologique est poussé par une contrainte organisationnelle (network-based technology, Le Bas et Zuscovitch, 1993). Les industries de transport ont pratiquement toutes une composante organisationnelle importante. C'est la firme Maître d'Euvre qui agence les savoirs et les technologies nécessaires en vue de la réalisation de leur objet industriel.

Une carte des trajectoires technologiques

\begin{tabular}{|c|c|c|c|c|}
\hline \multirow{3}{*}{$\begin{array}{l}\text { Intensité } \\
\text { en R-D }\end{array}$} & \multicolumn{2}{|c|}{$\begin{array}{l}\text { Produits plutôt } \\
\text { différenciés } \\
\text { et petites séries }\end{array}$} & \multicolumn{2}{|c|}{$\begin{array}{l}\text { Produits plutôt } \\
\text { banalisés } \\
\text { et grandes séries }\end{array}$} \\
\hline & \multicolumn{2}{|c|}{$\begin{array}{l}\text { offreurs } \\
\text { spécialisés }\end{array}$} & \multicolumn{2}{|c|}{$\begin{array}{c}\text { rendements croissants } \\
\text { à l'échelle }\end{array}$} \\
\hline & 1 & \multicolumn{2}{|c|}{$\begin{array}{c}\text { offreurs dominants } \\
2\end{array}$} & 4 \\
\hline Forte & \multicolumn{4}{|c|}{$\begin{array}{l}\text { à orientations scientifiques } \\
3\end{array}$} \\
\hline
\end{tabular}


Deux facteurs permettent de discriminer les trajectoires technologiques: l'intensité technologique (évaluée par l'investissement en R-D par unité de chiffre d'affaires) et la grandeur des séries associée au type de produit.

Lorsqu'on se situe au niveau des firmes, on doit suivre K. Pavitt et d'autres chercheurs du SPRU (Pavitt et al., 1989) qui ont proposé récemment de modifier cette typologie en introduisant une autre trajectoire dite "d'information intensive » ou la trajectoire à fort contenu d'information. Les progrès techniques permis par les technologies de l'information créent des opportunités pour améliorer, renouveler les logiciels. Il y a là d'importants gains de productivité attendus pour l'avenir. Une grande fraction du secteur des services pourrait supporter ce type de trajectoire. Toutefois, une firme peut suivre plus d'une trajectoire technologique, par exemple, une grande entreprise du secteur informatique construit des trajectoires relevant du renouvellement scientifique, d'une logique de rendements croissants et à fort contenu en information. Chacune des trajectoires implique un processus d'apprentissage différent. D'abord, parce que les sources de l'innovation se révèlent différentes: le laboratoire de R-D pour la trajectoire à renouvellement scientifique, les services d'ingénierie pour les trajectoires à rendements d'échelle, le producteur d'équipement lorsque l'innovation est achetée, etc.

Dans le groupe d'entreprises, trois grandes trajectoires sont représentées:

- Les trajectoires à fondement scientifique: les petites entreprises de la branche des textiles qui ont été étudiées sont assez représentatives de cette trajectoire: forte intensité technologique (l'investissement en R-D est élevé, il sert à concevoir des produits nouveaux incorporant des matériaux nouveaux - les textiles techniques - ou un autre design). C'est plutôt en amont au niveau de la filature que l'on trouve les grandes entreprises exploitant des trajectoires technologiques à échelle intensive. Bien évidemment, d'autres moyennes entreprises industrielles développent d'autres types de trajectoires.

- La trajectoire "offreurs spécialisés» peut être assez représentative de ce que l'on a rencontré dans la mécanique (mais peut-être pas dans toute la mécanique). Les firmes recherchent l'amélioration de l'efficience de leurs produits, la qualité des dispositifs, leur sûreté, etc. Elles doivent également bien cerner les besoins des demandeurs, ce qui conduit souvent à rechercher des segments de marché pour lesquels elles sont en position de monopole (niches).

- La trajectoire "offreurs dominants» se caractérise par le fait que les offreurs d'équipement ou d'autres inputs contrôlent la technologie, connaissent les utilisateurs et «dominent» les relations clients- 
fournisseurs. La difficulté pour le producteur est de faire émerger les savoir-faire nécessaires, ce qui prend du temps, ou de capter, via le fournisseur d'équipement ou d'input, l'expérience des concurrents. Si les entreprises de la construction électronique développent ce type de trajectoire, on la rencontre parfois également dans le textile. Il est aussi vrai que des firmes exploitant cette trajectoire possèdent des configurations techniques et organisationnelles qui les rapprochent d'une trajectoire «à échelle intensive» (machines ou lignes de fabrication fonctionnant avec de très grandes séries).

\section{Le recours aux ressources technologiques externes}

On distingue assez traditionnellement deux catégories d'acquisition externe de ressources technologiques (toutes les deux coûteuses):

1. L'acquisition formalisée et marchande de technologies qui prend plusieurs voies:

(Dussauge et Ramanantsoa, 1987)

- achat de matériel ou d'équipement,

- contrat d'assistance technique (maintenance),

- embauche de personnel pour leur compétence technique,

- achat de brevets, licences,

- contrat de recherche technique (centre technique, laboratoire, autres entreprises),

- contrat de recherche appliquée (université, centre de R-D...),

- rachat (éducatif) d'entreprises (ou coopérations interentreprises).

On a pu montrer que ces différentes voies sont inégalement risquées, mais aussi marquées par des temps différents de maîtrise des technologies. C'est pour cette raison que les petites entreprises de haute technologie préferent la génération interne de la technologie, ou lorsqu'elle procède à une acquisition (externe), c'est bien souvent pour renforcer leur R-D interne (Oakey et al., 1987).

2. L'acquisition non formalisée d'informations techniques souvent non marchandes :

(Kelley et Brooks, 1991):

- lecture de médias écrits et de brochures techniques, 
- rapports techniques avec les fournisseurs d'équipement à l'occasion des relations achats/ventes,

- autres contacts qu'avec les fournisseurs (syndicats professionnels...),

- participation à des salons, conférences, séminaires,

- dialogue (avec partage de l'information) avec les firmes clientes ou les fournisseurs (en dehors des relations achats/ventes).

Ce sont ces différentes voies d'accès (externes) aux technologies qui ont été retenues pour interroger les entrepreneurs de notre groupe quant à leur stratégie technologique. Toutefois, aucun rachat éducatif d'entreprises n'a été recensé ni d'accord interentreprises.

Dans ce travail, nous rappellerons d'abord la prédiction essentielle qui émerge de la littérature: les PME se différencient principalement par ces stratégies différentes d'accès aux ressources externes. Une première évaluation des stratégies d'acquisition à partir des données recueillies nous conduira, ensuite, à décrire deux modèles d'apprentissage. Enfin, une analyse plus clinique permettra de mettre en évidence les freins et les limites de l'acquisition externe de la technologie.

\subsection{Une conclusion des études sur les stratégies technologiques des petites entreprises}

C'est la forme du recours aux compétences externes plus que son ampleur qui explique l'engagement de la firme dans un processus positif de changement technique.

Nous nous référons ici au travail de M.R. Kelley et H. Brooks (1991). Bien que cette étude ne porte que sur les petites entreprises, il a semblé intéressant de retenir les conclusions de ce travail afin de voir si elles pouvaient éventuellement s'appliquer à nos MEI.

La problématique des ressources technologiques externes a une signification particulière pour les PME/PMI. On pense en effet que le manque de connaissances externes qui empêche la firme de s'engager dans un processus d'apprentissage de nouvelles technologies, notamment parce que les petites entreprises n'ont pas la taille critique minimum, pourrait être comblé par l'appel aux ressources externes afin de suppléer aux limites des ressources internes. En réalité, il a été montré (Kelley et Brooks, 1991) que c'est le type de recours aux ressources externes qui est déterminant et beaucoup moins l'ampleur de l'achat de compétences. Le travail de M.R. Kelley et H. Brooks fait apparaître deux configurations polaires dans le comportement d'achats de compétences 
externes, selon le type de liaisons que la firme entretient avec son environnement (liaisons passives ou liaisons actives).

Les formes de recours aux ressources technologiques externes (M.R. Kelley et H. Brooks)

\begin{tabular}{|c|c|c|c|}
\hline $\begin{array}{c}\text { Type } \\
\text { de liaisons }\end{array}$ & $\begin{array}{c}\text { Support } \\
\text { de la liaison } \\
\text { entre la firme } \\
\text { et son environnement }\end{array}$ & $\begin{array}{c}\text { Capacité } \\
\text { de recherche } \\
\text { de l'organisation }\end{array}$ & $\begin{array}{c}\text { Acteur dominant } \\
\text { dans l'interaction } \\
\text { entreprise/ } \\
\text { environnement }\end{array}$ \\
\hline $\begin{array}{c}\text { Liaisons } \\
\text { passives et } \\
\text { plutôt asociales }\end{array}$ & $\begin{array}{c}\text { Lecture } \\
\text { de médias écrits } \\
\text { (brochures techniques } \\
\text { Et rapports techniques } \\
\text { dans les relations } \\
\text { achats/ventes avec } \\
\text { leurs fournisseurs } \\
\text { d'équipements }\end{array}$ & $\begin{array}{l}\text { Faible } \\
\text { Ce sont les agents } \\
\text { extérieurs qui vont } \\
\text { vers la firme qui } \\
\text { possède finalement } \\
\text { une faible capacité } \\
\text { d'apprentissage }\end{array}$ & L'environnement \\
\hline $\begin{array}{l}\text { Liaisons actives } \\
\text { et socialisées }\end{array}$ & $\begin{array}{l}\text { En plus des supports } \\
\text { déjà indiqués, } \\
\text { les contacts } \\
\text { ne se font pas qu'avec } \\
\text { les fournisseurs. } \\
\text { Il y a partage } \\
\text { de l'information } \\
\text { Assistance à des salons, } \\
\text { séminaires... }\end{array}$ & $\begin{array}{c}\text { Forte } \\
\text { C'est la firme } \\
\text { qui va contacter } \\
\text { les autres agents }\end{array}$ & L'agent \\
\hline
\end{tabular}

La conclusion serait que la petite firme qui n'a que des liaisons passives (et asociales) avec les producteurs d'équipement ou la clientèle ne s'engagera que difficilement dans un processus maîtrisé de construction de nouvelles techniques d'une certaine ampleur, aussi longtemps qu'une menace crédible venant de nouveaux entrants ne constitue un réel danger. Sa dynamique d'apprentissage technologique (externe) est faible.

Il y aurait un danger à concevoir la politique technologique comme devant accroître les efforts en faveur de l'assistance aux petites entreprises en ignorant l'existence, et donc également les effets, des relations passives de la firme avec son environnement. Si l'on considère que seules les liaisons actives fondées sur une collaboration dense (active) permettent l'engagement de la firme dans un processus vertueux d'acquisition de compétence technologique, alors la création de liaisons fortes entre la firme et son environnement devrait être un objectif des politiques technologiques publiques. 


\subsection{Le recours aux ressources technologiques externes: une évaluation globable à partir des données recueillies sur l'échantillon de MEI}

Les entreprises recourent à des ressources technologiques externes sous des formes différentes. En premier lieu, il y a des investissements technologiques externes plus fréquemment réalisés que d'autres. L'accès privilégié aux technologies (externes) est l'achat d'équipement, l'embauche de personnel (sans doute en vue de posséder des compétences techniques pointues), des contrats de recherche technique (par exemple, avec des centres techniques). L'acquisition de brevets est, par opposition, un mode d'accès très peu utilisé (seulement par $22 \%$ des entreprises, soit 4 entreprises du groupe). Enfin, moins d'une entreprise sur deux s'en remet à une entreprise extérieure pour assurer la maintenance de ses équipements techniques sur lesquels sont basés ses processus de fabrication.

\section{Type d'acquisition technologique}

Pourcentage d'entreprises ayant recours à ce type d'acquisition

\begin{tabular}{ll}
\hline Achat de matériel & $82 \%$ \\
Embauche de personnel & $59 \%$ \\
Contrat de recherche technique & $53 \%$ \\
Contrat d'assistance technique & $47 \%$ \\
Contrat de recherche appliquée & $24 \%$ \\
Achat de brevet & $12 \%$
\end{tabular}

Mode de collecte de l'information technique

Lecture de médias

Participation à des salons, conférences

Rapports techniques avec les fournisseurs d'équipement

Partage de l'information (clients, fournisseurs)

Autres contacts
Pourcentage d'entreprises ayant recours à ce type d'acquisition
$82 \%$

$82 \%$

$71 \%$

$65 \%$

$53 \%$

Les facteurs qui rendent compte de ces comportements d'investissements technologiques externes sont sûrement déterminés par les caractéristiques structurelles des secteurs. S'agissant maintenant de l'acquisition d'informations technologiques sous des aspects moins formalisés, les firmes s'engagent dans des recherches d'informations variées avec des fréquences plus fortes que les petites entreprises (Kelley et Brooks, 1991). Les moyens très majoritairement utilisés sont la lecture de médias, la participation à des salons ou 
des conférences. L'interactivité technique avec les fournisseurs est aussi un moyen d'acquérir des informations, mais les firmes semblent avoir plus de difficulté à «partager» les informations avec leurs clients ou leurs fournisseurs.

\subsection{Les formes du recours aux ressources technologiques externes diffèrent selon les entreprises: l'émergence de deux modèles d'apprentissage}

Il est délicat de retrouver les catégories polaires de M.R. Kelley et H. Brooks (1991); les firmes qui ont des liaisons passives, plutôt «asociales » avec leur environnement, et les firmes qui ont des relations plus sociales et qui sont donc plus actives. Ceci s'explique certainement par le fait que nous n'avons pas affaire à des petites entreprises, mais plutôt à des moyennes. Toutefois, il s'avère qu'à l'intérieur de notre échantillon, les firmes n'ont pas le même degré d'engagement ou d'interactivité avec leur environnement. Certaines ont un spectre d'interactivité plus large, établissent plus d'échanges avec leur environnement. Ainsi, nous pouvons distinguer deux types d'apprentissage:

- Un processus d'apprentissage complexe, dense, mené par des entreprises plus (mieux) structurées. En termes d'apprentissage technologique, ces entreprises vont jusqu'à la sous-traitance de la R-D et construisent des liaisons avec des laboratoires. En termes d'apprentissage commercial, elles réalisent une bonne adéquation entre leurs technologies, leurs produits, leurs marchés (ainsi, elles développent souvent des politiques de niches). On serait tenté de dire qu'elles réalisent une bonne absorption technologique sur une base large.

- Un processus d'apprentissage simple mené par des entreprises qui apparaissent beaucoup moins structurées. En termes d'apprentissage technologique externe, ces entreprises ont un accès très particulier aux ressources technologiques externes, il passe exclusivement par l'achat d'équipement, de l'assistance technique ou par le recrutement de personnel pour ses compétences techniques. Pas d'achat de technologies sous forme de brevet, aucun achat du produit de la recherche (cependant, elles peuvent parfois sous-traiter des études à des centres techniques).

Nous désignons par P1 la population d'entreprises développant le premier type d'apprentissage, par P2 la population qui fait appel au second type d'apprentissage.

Les formes du recours aux ressources technologiques externes diffèrent entre les deux populations. Pour ce qui concerne l'investissement technologique 
externe, la différence essentielle tient dans la stratégie de sous-traitance de la $\mathrm{R}-\mathrm{D}$. Certaines entreprises moyennes n'ont pas de stratégie de sous-traitance de $R-D$ appliquée, par ailleurs, elles ne développent que très peu de relations avec des centres techniques ou des laboratoires (population 2). De plus, elles ne procèdent jamais à l'achat de brevet ou de licence.

\section{Acquisition formalisée de technologies}

\begin{tabular}{lcc}
\hline \multicolumn{1}{c}{$\begin{array}{c}\text { Type d'acquisition } \\
\text { technologique }\end{array}$} & $\begin{array}{c}\text { Pourcentage } \\
\text { d'entreprises } \\
\text { de la population 1 } \\
\text { ayant recours } \\
\text { à ce type d'acquisition }\end{array}$ & $\begin{array}{c}\text { Pourcentage } \\
\text { d'entreprises } \\
\text { de la population 2 } \\
\text { ayant recours } \\
\text { à ce type d'acquisition }\end{array}$ \\
\hline Achat de matériel & $80 \%$ & $86 \%$ \\
Contrat d'assistance technique & $50 \%$ & $42 \%$ \\
Embauche de personnel & $60 \%$ & $57 \%$ \\
Contrat de recherche technique & $80 \%$ & $14 \%$ \\
Contrat de recherche appliqué & $40 \%$ & 0 \\
Achat de brevet & $20 \%$ & 0 \\
\hline
\end{tabular}

En ce qui a trait aux canaux d'accès à l'information technique, on trouve dans chacune des populations représentées tous les modes d'accès à l'information. Si l'on classe pour chacune des populations les modes de collecte des informations selon l'ordre de fréquence des plus grandes aux plus faibles, on ne constate pas de grandes différences entre les deux populations. La différence entre les deux tient sans doute au fait que pour un mode d'accès à l'information donné, la fréquence du recours est plus grande pour la population 1 que pour la population 2 (à l'exception des stratégies de partage de l'information).

\begin{tabular}{lcc}
\hline $\begin{array}{c}\text { Mode de collecte de } \\
\text { l'information } \\
\text { technique }\end{array}$ & $\begin{array}{c}\text { Pourcentage } \\
\text { d'entreprises } \\
\text { de la population } \\
\text { ayant recours } \\
\text { à ce type d'acquisition }\end{array}$ & $\begin{array}{c}\text { Pourcentage } \\
\text { d'entreprises } \\
\text { de la population 2 } \\
\text { ayant recours } \\
\text { à ce type d'acquisition }\end{array}$ \\
\hline $\begin{array}{l}\text { Lecture de médias } \\
\text { Participation à des salons, conférences }\end{array}$ & $90 \%$ & $71 \%$ \\
$\begin{array}{l}\text { Rapports techniques } \\
\text { avec les fournisseurs d'équipement }\end{array}$ & $90 \%$ & $71 \%$ \\
$\begin{array}{l}\text { Partage de l'information } \\
\text { (clients, fournisseurs) }\end{array}$ & $60 \%$ & $57 \%$ \\
Autres contacts & $50 \%$ & $71 \%$ \\
\hline
\end{tabular}


Les trajectoires technologiques pratiquées et les structures des entreprises expliquent en (grande) partie l'engagement des entreprises dans deux sentiers d'apprentissage (les deux facteurs sont bien évidemment corrélés, si ce n'est totalement associés) beaucoup mieux l'appartenance sectorielle.

Il est à remarquer que les firmes de la population 1 ont toutes bénéficié d'un crédit impôt-recherche, ce qui peut être interprété de la façon suivante: ces entreprises ont une bonne "gestion» de leur fonction de R-D; elles en ont également une bonne évaluation économique et connaissent les dispositifs publics d'aide à l'investissement en R-D. Par ailleurs, il n'est pas étonnant qu'elles seules puissent également se lancer dans la sous-traitance de R-D (ce qui corrobore les travaux de C. Mowery, 1983, quant à la liaison entre processus de R-D interne et sous-traitance externe de R-D). Les firmes de P1 sont en général plus grandes, elles ont une intensité technologique plus élevée, possèdent un réel service de recherche identifié et structuré. Les deux populations se caractérisent par une structuration différente de la R-D et du développement technologique (forte pour la population 1 et quasi inexistante pour la population 2), par une intensité technologique élevée pour la population 1, quasi nulle pour la population 2. Les entreprises de P1 sont mieux organisées et investissent plus de moyens en R-D. On trouve ainsi dans la population 1 les firmes à trajectoire technologique fondée sur les découvertes scientifiques (dites «à orientations scientifiques»), alors que les firmes développant une trajectoire d'offreurs spécialisés se retrouvent plutôt dans la population 2.

\section{Les caractéristiques des deux sous-populations}

\begin{tabular}{lcc}
\hline & POPULATION 1 & POPULATION 2 \\
\hline Taille moyenne (effectif) & 218 & 170 \\
Dispersion de la taille & $\begin{array}{c}\text { Seule une entreprise } \\
\text { a un effectif supérieur } \\
\text { à } 180 \text { personnes }\end{array}$ & $\begin{array}{c}4 \text { entreprises } \\
\text { ont un effectif supérieur } \\
\text { à } 210 \text { personnes }\end{array}$ \\
$\begin{array}{l}\text { Intensité technologique } \\
\text { moyenne (R-D/CA) }\end{array}$ & $4,0 \%$ & $0,2 \%$ \\
$\begin{array}{l}\text { Variété sectorielle } \\
\text { (n) entreprises } \\
\text { du textile }\end{array}$ & $\begin{array}{c}4 \text { entreprises } \\
\text { de la mécanique } \\
3 \text { entreprises }\end{array}$ \\
$\begin{array}{c}\text { Entreprises } \\
\text { un la mécanique }\end{array}$ & $\begin{array}{c}\text { de la construction } \\
\text { électronique }\end{array}$ \\
$\begin{array}{c}\text { Entreprises possédant } \\
\text { un service de recherche }\end{array}$ & $75 \%$ & $25 \%$
\end{tabular}


Les structures d'organisation des firmes et les trajectoires technologiques pratiquées expliquent la division en deux sous-populations.

\subsection{Freins et limites de l'acquisition technologique externe: un aperçu "clinique»}

Afin d'avoir une vision assez fidèle des contraintes et des freins auxquels sont soumises les entreprises lorsqu'elles construisent des relations d'acquisitions externes de technologie, il a été demandé aux personnes interviewées d'identifier les principales difficultés parmi quatre grands ensembles : culturels, financiers, en termes de ressources humaines, techniques. Il n'était ni demandé de classer ces facteurs selon leur importance, ni d'imputer la cause des difficultés à la firme, à l'agent avec lequel l'entreprise construisait sa relation ou à l'environnement. Toutefois, des critiques ont pu être formulées à l'encontre des sociétés de conseil (qui ont mal présenté les plans d'investissements) ou des fournisseurs d'intrants technologiques (qui ne respectaient pas les délais). Signalons maintenant les problèmes.

En ce qui concerne le «domaine culturel», les réponses vont d'un constat banal (et très terre à terre), «les ingénieurs parlent mal anglais », à des remarques plus cruciales sur les différences d'approches et de mentalités entre les industriels et les chercheurs des organismes publics qui hypothèquent bien souvent les relations nouées entre les entreprises et les laboratoires publics (censés peu connaître les produits et les contraintes industrielles...). Il y a là un problème plus général lié à la difficulté pour les MEI de construire des stratégies de réseaux dans le domaine de la recherche. Pour ce qui est de l'aspect financier, des MEI déclarent ne pas rencontrer de problèmes et même disposer d'un «rapport de forces favorable dans ce domaine». Toutefois, certaines avancent bien l'insuffisance de ressources, mais ce n'est pas toujours le facteur qui bloque l'investissement technologique externe. Il y a en revanche quasi-unanimité en ce qui concerne les ressources humaines: le manque de personnel qualifié (en production, au niveau des agents de maîtrise, comme au plan de certaines spécialités techniques...) est toujours avancé; de même que la nécessaire «réformation» de la main-d'œuvre. Ce constat émis, le diagnostic pourrait être qu'il s'agit souvent de vieilles industries (textile, forge, fonderie) pour lesquelles les savoir-faire proviennent d'une patiente accumulation d'expérience, le recours au marché externe du travail ne remplace pas les compétences acquises sur le tas. Les facteurs techniques sont toujours mis en avant par toutes les entreprises. Les personnes interviewées décèlent en réalité les principaux dysfonctionnements de l'apprentissage technologique externe: la mise au point des fabrications sur des machines spéciales prend du temps, les démarrages de 
production sont difficiles (et coûteux : voir le tableau, page suivante), l'assimilation des résultats ne se fait pas sans problème, la maîtrise des procédés (nouveaux) reste encore incertaine... Souvent l'entreprise met les difficultés sur le compte des entre-prises extérieures ( il n'y a pas toujours un bon suivi d'assistance technique») ou à son propre débit («le suivi de qualité pourrait être meilleur»). Une entreprise signale une autre difficulté de l'apprentissage: les obstacles à l'intégration des résultats concernant les nouveaux procédés aux recherches de l'entreprise. Quelquefois, c'est l'interaction entre l'entreprise et les firmes qui fournissent l'information ou l'intrant technologique qui pose problème, puisque les firmes désirent avant tout conserver leur savoir-faire empirique, éviter que d'autres entreprises (sous-entendues concurrentes) s'approprient leurs résultats. Cette «obligation de confidentialité » (jugée à la base de l'avantage concurrentiel de l'entreprise) «parasite» les relations avec les autres partenaires autour d'un projet technologique.

Afin d'avoir une vision plus juste des difficultés de l'apprentissage technologique externe, on a demandé aux MEI enquêtées de décrire un projet technologique (pour lequel sont intervenus des agents extérieurs) en notant les difficultés et les freins, mais aussi les aides et les appuis possibles. Une conclusion émerge : les MEI semblent vouloir compter sur leurs propres compétences et sont plutôt sceptiques quant à l'efficacité des «appuis extérieurs».

\section{En guise de conclusion: stratégies et politiques technologiques}

Nous résumons maintenant les principaux points qui ressortent de ce travail:

1. Les stratégies d'innovation, de création technologique ou d'absorption technologique sont très intimement liées aux marchés sur lesquels les entreprises interviennent. Ce point, désormais acquis dans la littérature (Docter et Stockman, 1987), signifie que les entreprises pensent en même temps les rapports entre technologies-produits-marchés. Les MEI qui réalisent le meilleur apprentissage sont celles qui conçoivent leur investissement d'acquisition externe de technologies en liaison avec une stratégie de marché. Il n'y a donc pas de voie royale pour maîtriser les ressources technologiques externes et les incorporer aux compétences externes, mais autant de façons que de trajectoires technologiques, de structures d'entreprises, de cultures d'organisation... 


\section{Quatre entreprises : quatre visions des difficultés d'un projet d'investissement technologique}

\begin{tabular}{|c|c|c|c|c|}
\hline & $\begin{array}{l}\text { Entreprise } \\
\text { de mécanique }\end{array}$ & $\begin{array}{l}\text { Entreprise } \\
\text { de mécanique }\end{array}$ & $\begin{array}{l}\text { Entreprise } \\
\text { de textile }\end{array}$ & $\begin{array}{l}\text { Entreprise } \\
\text { de textile }\end{array}$ \\
\hline $\begin{array}{l}\text { Les } \\
\text { difficultés } \\
\text { du projet }\end{array}$ & $\begin{array}{l}\text { Elles résident dans } \\
\text { les relations avec } \\
\text { les fournisseurs. } \\
\text { Peu de fournisseurs } \\
\text { maîtrise bien } \\
\text { la maintenance du } \\
\text { matériel ou le SAV. }\end{array}$ & $\begin{array}{l}\text { Le métier se } \\
\text { transforme, } \\
\text { ce qui nécessite } \\
\text { la création } \\
\text { de compétences } \\
\text { techniques } \\
\text { (bureau d'étude). }\end{array}$ & $\begin{array}{l}\text { C'est le service } \\
\text { recherche } \\
\text { qui réalise } \\
\text { l'innovation } \\
\text { (des produits } \\
\text { nouveaux } \\
\text { essentiellement). }\end{array}$ & $\begin{array}{l}\text { L'entreprise a } \\
\text { ciblé ses besoins } \\
\text { en recherche en } \\
\text { ce qui concerne } \\
\text { les nouvelles } \\
\text { matières } \\
\text { premières. }\end{array}$ \\
\hline $\begin{array}{l}\text { Les } \\
\text { freins } \\
\text { rencontrés }\end{array}$ & $\begin{array}{l}\text { Les fournisseurs } \\
\text { sont les principaux } \\
\text { freins, car ils ne } \\
\text { veulent vendre que } \\
\text { des produits } \\
\text { standard. Ils sont } \\
\text { plus commerciaux } \\
\text { que techniciens. }\end{array}$ & $\begin{array}{l}\text { S'il y a des aides } \\
\text { extérieures pour } \\
\text { l'informatique, } \\
\text { il n'y a pas d'aides } \\
\text { pour les matériels } \\
\text { de production. }\end{array}$ & $\begin{array}{l}\text { Ils sont d'ordre } \\
\text { technique } \\
\text { essentiellement. }\end{array}$ & $\begin{array}{l}\text { C'est la contrainte } \\
\text { budgétaire } \\
\text { qui est forte. }\end{array}$ \\
\hline $\begin{array}{l}\text { Les } \\
\text { appuis } \\
\text { possibles }\end{array}$ & $\begin{array}{l}\text { Un consultant ne } \\
\text { servirait à rien. } \\
\text { L'entreprise } \\
\text { manque de } \\
\text { compétences sur } \\
\text { le vieillissement. }\end{array}$ & $\begin{array}{l}\text { Le syndicat } \\
\text { professionnel } \\
\text { assure la } \\
\text { normalisation, } \\
\text { mais pas d'autres } \\
\text { choses. }\end{array}$ & $\begin{array}{l}\text { L'expérience } \\
\text { constitue la base } \\
\text { d'un bon } \\
\text { apprentissage. }\end{array}$ & Pas de réponse. \\
\hline $\begin{array}{l}\text { L'aide } \\
\text { extérieure } \\
\text { attendue }\end{array}$ & Aucune. & $\begin{array}{l}\text { Éventuellement } \\
\text { contacts informels } \\
\text { au niveau régional. }\end{array}$ & Pas de réponse. & Pas de réponse. \\
\hline
\end{tabular}

2. Nous avons montré à partir de notre groupe d'une vingtaine d'entreprises qu'émergent deux modèles d'apprentissage:

- un processus d'apprentissage complexe, dense, mené par des entreprises plus (mieux) structurées. En termes d'apprentissage technologique, ces entreprises vont jusqu'à la sous-traitance de la $R-D$ et construisent des liaisons avec des laboratoires. En termes d'apprentissage commercial, elles réalisent une bonne adéquation entre leurs technologies, leurs produits, leurs marchés (ainsi, elles développent souvent des politiques de niches). On serait tenté de dire qu'elles réalisent une bonne absorption technologique sur une base large.

- un processus d'apprentissage moins complexe (plus simple) mené par des entreprises qui apparaissent beaucoup moins structurées. En 
termes d'apprentissage technologique externe, ces entreprises ont un accès très particulier aux ressources technologiques externes: il passe exclusivement par l'achat d'équipement, par l'assistance technique ou par le recrutement de personnel pour ses compétences techniques. Pas d'achat de technologies sous forme de brevet, aucun achat du produit de la recherche (elles peuvent, toutefois, parfois sous-traiter des études à des centres techniques).

Désignons par P1 la population d'entreprises développant le premier type d'apprentissage, par P2 la population qui construit le second type d'apprentissage. Les firmes de P1 sont en général plus grandes, elles ont une intensité technologique plus élevée, possèdent un réel service de recherche. Les deux populations se caractérisent par une structuration différente de la R-D et du développement technologique (forte pour la population 1 et quasi inexistante pour la population 2), par une intensité technologique élevée pour la population 1 , quasi nulle pour la population 2. Les entreprises de P1 sont mieux organisées et investissent plus de moyens en R-D. On trouve ainsi dans la population 1 les firmes à trajectoire technologique fondée sur les découvertes scientifiques (dites «à orientations scientifiques»), alors que les firmes développant une trajectoire d'offreurs spécialisés se retrouvent plutôt dans la population 2.

Bien entendu, ces conclusions seront à confirmer sur un échantillon plus large et plus diversifié d'entreprises.

3. Notre recherche confirme dans ses grandes lignes la vision de S.J. Mantel et G. Rosegger (1987) quant à l'importance d'institutions tierces dans le processus de diffusion de l'innovation; celle de P.R. Beije et H.W. Buise (1990), selon laquelle un manque de relations technologiques denses (de type réseaux) peut diminuer l'efficacité des investissements technologiques des firmes. Notre étude réalisée auprès d'une vingtaine de moyennes entreprises industrielles révèle que la plupart des entreprises participent à un ou plusieurs réseaux (type réseaux industriels locaux, réseaux de compétence...). Précisément, au regard des moyennes entreprises industrielles, on retrouve ici deux populations de firmes:

- celles qui font suffisamment de R-D, qui disposent d'un bon niveau de compétences techniques internes et qui peuvent définir les stratégies technologiques externes et investissent dans des réseaux technologiques,

- celles qui ne font pas ou qui font peu de R-D, qui disposent de peu de compétences internes et ne recourent que de façon informelle à des informations technologiques externes; elles ne sont presque jamais immergées dans des réseaux d'échanges technologiques. 
Notre étude, dans le prolongement de leur travail, plaide largement pour une politique de consolidation/densification des réseaux technologiques en faveur des moyennes entreprises industrielles, en particulier les réseaux mettant en relation les MEI et les centres de recherche. Si nous mettons l'accent sur le phénomène de réseaux, nous ne négligeons pas pour autant l'importance de la culture technico-économique du dirigeant (ou du cercle dirigeant) de l'entreprise. On sait que dans la PME, l'activité inventive reste fortement personnalisée. Or, les réseaux technologiques sont eux aussi soutenus par quelques personnes. Cela signifie que le système d'emploi des MEI et la politique d'embauche sont des moyens d'accroître le savoir-faire de l'entreprise et donc, la capacité d'apprentissage technologique.

\section{Bibliographie}

ADLER, P.A. (1990), « Shared learning» dans Management Science, vol. 36, p. 938-957.

AOKI, M. (1990), «Towards an economic model of the Japanese firm», dans Journal of Economic Litterature, mars.

ARGYRIS, C. et D. SCHON (1978), Organisational Learning, Londres, Addison-Wesley.

BEIJE, P.R. et H.W. BUISE (1990), «Innovation policy for small and medium sized enterprises cost approach or network approach?» dans J. Allesch (dir.), Consulting in Innovation, Amsterdam, Elsevier.

CARLSON, B. et R. STANKIEWIEZ (1991), « On the nature, functions and composition of technological systems », Journal of Evolutionary Economics, vol. 1, p. 93-118.

Chesnais, F. (1988), «Les accords de coopération technique entre firmes indépendantes », STI Revue, $n^{\circ} 4$, OCDE.

COHEN, W.M. et D.A. LeVINTHAL (1990), « Absoptive capacity : a new perspective on learning and innovation », Administrative Science Quaterly, vol. 35, p. 128-152.

CYERT, R.M. et J.G. MARCH (1963), A Behavioural Theory of the Firm, Englewood Cliffs, N.J., Prentice-Hall.

DOCTER, J. et C. STOCKMAN (1987), «Innovation strategies of small industrial companies » dans R. Rothwell et J. Bessant (dir.), Innovation: Adaptation and Growth, Amsterdam, Elsevier.

DodGson, M. (dir.) (1989), Technology Strategy and the Firm: Management and Public Policy, Londres, Longman. 
Dodgson, M. (1991), «Technology learning, technology strategies and competitive pressures », British Journal of Management, vol. 2, p. 133-149.

Dodgson, M. (1991), The Management of Technological Learning, Berlin et New York, W. De Gruyter.

Dussauge, P. et L. Ramanantsoa (1987), Technologie et stratégie d'entreprise, McGraw-Hill.

GAFFARD, J.L. (1990), Économie industrielle et de l'innovation, Paris, Dalloz.

GÉNIAUX, I. (1992), «Enquête exploratoire : les PME dans les échanges technologiques », Rapport de recherche ESC Lyon.

JULIEN, P.A. et M. MARSCHESNAY (1992), « Des procédures aux processus stratégiques en PME», Piccola Impresa, $\mathrm{n}^{\circ}$ 1, Turin, p. 13-41.

KatZ, D. et R.L. KaHn (1966), The Social Psychology of Organizations, New York, Wiley.

KELLEY, M.R. et H. BROOKS (1991), « External learning opportunities and the diffusion of process innovations to small firms », Technological Forecasting and Social Change, vol. 39, p. 103-125.

LE BAS, C. (1989), «La stratégie d'automatisation de la PME : réflexions à partir d'études de cas », Économies et Sociétés, Sciences de gestion, ${ }^{\circ} 13$, p. 165-185.

LE BAS, C. et E. ZuSCOVITCH (1993), « Apprentissage technologique et organisation: une analyse des configurations micro-économiques », Economies et Sociétés, Série Dynamique technologique et Organisation.

MANSFIELD, E. (1968), Industrial Research and Technological Innovation, New York, W.W. Norton.

MANTEL, S.J. et G. Rosegger (1987), «The role of third-parties in the diffusion of innovations : a survey ", dans R. Rothwell et J. Bessant (dir.), op. cit.

MOWERY, D.C. (1983), «The relationship between contractual and intrafirm forms of industrial research in manufacturing 1900-1940 », Exploration in Economic History, p. 351-374.

NELSON, R. et S. WINTER (1982), An Evolutionary Theory of Economic Change, Cambridge, Mass., Belknap Press of Harvard University.

OAKEY, R.P. et al . (1987), « Research and development and competitive performance in British and American high technology small firms », dans R. Rothwell et J. Bessant (dir.), op. cit.

OCDE, (1992), La Technologie et l'Économie, Rapport TEP, Paris, OCDE.

PAVITT, K. (1984), «Sectoral patterns of technical change : towards a taxonomy and a theory » Research Policy, vol. 13. 
POWELL, W.W. (1990), « Neither market nor hierarchy: network forms of organization ", dans B.N. Straw et L.L. Cummings (dir. de recherche), Organization Behavior, vol. $12, \mathrm{n}^{\circ} 2$.

Rothwell, R. et J. Bessant (dir.) (1987), Innovation: Adaptation and Growth, Amsterdam, Elsevier.

Radnor, M. (1991), Technology Acquisition Strategies and Processes: A Reconsideration of the "Make Versus Buy» Decision, Londres, Longman.

Rosenberg, N. (1982), Inside the Black Box: Technology and Economics, Cambridge, Cambridge University Press.

Rosenberg, N. et S.J. KLINE (1986), «An overview of innovation » dans R. Landau et N. Rosenberg (dir.), The Positive Sum Strategy, National Academic Press.

SESSI-EAE (1991), L'enquête innovation, Paris.

SHARP, M. (1990), « Technological trajectories and corporate strategies in the diffusion of biotechnology », dans Technology and Investment Crucial Issues for the 1990s, OCDE et Londres, Pinter Publisher. 